\title{
Two Stage Portfolio Selection and Optimization Model with the Hybrid Particle Swarm Optimization
}

\author{
${ }^{1}$ Kashif Bin Zaheer*, ${ }^{2}$ Mohd Ismail Bin Abd Aziz, \\ ${ }^{3}$ Amber Nehan Kashif, ${ }^{4}$ Syed Muhammad Murshid Raza \\ ${ }^{1,2,3}$ Department of Mathematical Sciences, Faculty of Science, UTM. \\ 81310 UTM Johor Bahru, Malaysia \\ ${ }^{1,3,4}$ Department of Mathematical Sciences, Faculty of Science, FUUAST, Karachi, Pakistan. \\ ${ }^{*}$ Corresponding author: kbzaheer@gmail.com.
}

Article history

Received: 31 July 2017

Received in revised form: 15 October 2017

Accepted: 19 October 2017

Published on line: 1 June 2018

\begin{abstract}
The selection criteria play an important role in the portfolio optimization using any ratio model. In this paper, the authors have considered the mean return as profit and variance of return as risk on the asset return as selection criteria, as the first stage to optimize the selected portfolio. Furthermore, the sharp ratio (SR) has been considered to be the optimization ratio model. In this regard, the historical data taken from Shanghai Stock Exchange (SSE) has been considered. A metaheuristic technique has been developed, with financial tool box available in MATLAB and the particle swarm optimization (PSO) algorithm. Hence, called as the hybrid particle swarm optimization (HPSO) or can also be called as financial tool box particle swarm optimization (FTBPSO). In this model, the budgets as constraint, where as two different models i.e. with and without short sale, have been considered. The obtained results have been compared with the existing literature and the proposed technique is found to be optimum and better in terms of profit.
\end{abstract}

Keywords Portfolio Optimization; Profit; Risk; Shanghai Stock Exchange (SSE); Hybrid particle swarm optimization (HPSO); Financial tool box particle swarm optimization (FTB-PSO); Sharp ratio (SR); Budget; Short Sale.

Mathematics Subject Classification 46N60, 92B99.

\section{Introduction}

One of the attractions in the modern portfolio theory (MPT) is the portfolio optimization. The goal of such studies is to maximize the profit and minimize the risk. Optimization of the profit and risk can be dealt under the modern portfolio theory, introduced by Markowitz [1]. The Markowitz has treated the portfolio variance (risk) as one of the objective functions for the portfolio of assets, where as, he has treated the portfolio mean (profit) as the constraint in 
his mean-variance model [1]. In recent past Thirimanna et al. [2] have discussed the portfolio selection criteria using the two different techniques, cointegration and MPT, also the superior portfolios and strategy have been obtained through comparison of the sharp ratio (SR), information ratio (IR), return and risk in terms of performance. They have used the historical prices data from the Colombo Stock Exchange (CSE). Also, Tan and Lim [3] have discussed the performance of the selection of different types of universal portfolios (i.e. Helmbold and chi-square divergence universal portfolios) using the best current-run parameter technique extracted from mixture-current-run parameter of universal portfolio. They have selected the data set from local stock exchange. Chen et al. [4] have studied the probability distribution of returns for index prices of FTSE bursa Malaysia KLCI, using statistical (Heston) model and estimation (Simulated Maximum Likelihood) technique. They have used the Euler-Maruyama method to obtain the approximate solutions of the stochastic differential equation. According to them, investors could be able to plan their investments based on the results obtained. Sagir and Sathasivam [5] have taken into account the prices for stock market forecasting with an implementation of artificial neural network (ANN) and multiple linear regressions. Furthermore, other researchers [6-16] have also carried out the research with the mean-variance model, in different combination of various techniques.

Sharp [17] has worked on the portfolio optimization problem, he has established a relationship between mean and variance of the portfolio, known as sharp ratio (SR), as a single objective function with some other constraints or alone. The SR needs to be maximized in order to get the optimal solution of the portfolio [17]. Some other researchers [18-24] have also focused on SR, but in different ways.

Some researchers have developed the meta-heuristic techniques, because of the complexity of the models in the MPT. Few of them are genetic algorithms (GA), tabu search, particle swarm optimization(PSO), ant colony optimization(ACO), artificial bee colony (ABC) optimization, cuckoo search optimization and simulated annealing etc. [15] and the references therein. The PSO is also one of the meta-heuristic techniques used to optimize the portfolio [25,26]. It is a nature inspired technique, captures the behaviour of the bird flocking or fish schooling $[27,28]$. It is simple in application to the real world problems, like the portfolio selection and optimization $[29,30]$. It usually doesn't stick in the local optimum and hence easily reaches to global optimum. Because of this property it is considered as very efficient and effective in the portfolio selection and optimization problems $[8,31,32]$.

The literature review reveals that the researchers have paid a lot attention to the selection criteria and optimization of the portfolios. Due to the higher risk and intractability of stock prices, the profitable investment has become tricky. In this paper, the authors have considered the historical daily adjusted prices for the assets, taken from the Shanghai Stock Exchange 50 Index (SSE 50 Index) from 1st May, 2009 to 3rd April, 2009 i.e. for 21 days. Here, the reverse order of the dates has been mentioned same as from yahoo finance [25]. Having consideration to all, the focus of the study has become to obtain an optimal portfolio selection and solution. To obtain the optimum results from this model a hybrid algorithm, consisting of the financial tool box (FTB) and PSO needs to be developed. It would be the blend of basic FTB in MATLAB and the fundamental PSO, hence can be said as FTB-PSO.

Crux of this study would be to develop the simple models for selection and optimization of stocks portfolio, also, the development of the algorithm to obtain solutions of these models. The results show the combination of aforementioned techniques are helpful to obtains the good 
optimization strategy and quickly converges to the optimum solution. Decisively, the two stage portfolio selection and optimization model has been developed. Definitely, this research will contribute extensively for an individual investor, in the financial market, institutions and banks etc.

\section{Model for Portfolio Optimization (PO)}

Diversification plays a crucial role in portfolio optimization; it avoids the risk and attracts the return. For this purpose, lot of researchers have already worked on the Markowitz meanvariance model, the same as a single objective function and the SR with the combination of mean and variance as an efficient frontier. One of them is presented below [17,25].

\subsection{Sharpe Ratio (SR) Model}

One of the combination of mean-variance model is the SR model. It combines the mean and variance of the portfolio. It is used to evaluate the performance of the portfolio, also it adjusts the risk-adjusted measure of mean return [25]. Mathematically:

$$
\begin{gathered}
\mathrm{SR}=\frac{R_{p}-R_{f}}{\operatorname{Std} \operatorname{Dev}(p)} \\
R_{p}=\sum_{i=1}^{N} w_{i} r_{i} \\
\operatorname{StdDev}(p)=\sum_{i=1}^{N} \sum_{j=1}^{N} w_{i} w_{j} \sigma_{i j}
\end{gathered}
$$

where $p$ is the portfolio, $r_{i}$ is the return of the asset $i, w_{i}$ is the proportion invested in the asset $i, R_{p}$ is the mean return of the portfolio $p, R_{f}$ is the risk free rate of return for assets, $\sigma_{i j}$ is the covariance of the return for assets $i$ and $j, \operatorname{StdDev}(p)$ is the standard deviation of the returns for the portfolio $p$. Sharp ratio maximizes the mean return and minimizes the variance of return for the portfolio simultaneously, with the adjustment of the weights $w_{i}$.

\section{Models for Assets/Securities Selection}

It is important to define and explain the model for selection criteria of assets in the portfolio for two stage portfolio selection and optimization. The authors have considered the mean of the assets return as gain (profit) as given(4) and variance of the assets return as loss (risk) as given in(5), as an individual criteria to select the assets from the data set. Sorting of the data sets has been done with respect to the maximum profit of the assets in the descending order where as minimum risk of the assets has been done in the ascending order and then the desired number of assets have been selected. Mathematically the models are:

$$
\begin{array}{cc}
\text { Descend } \quad \overline{\mathrm{R}}_{i}, & i=1, \ldots, N \\
\text { Ascend } \quad \mathrm{V}_{\mathrm{R}}, & i=1, \ldots, N \\
\overline{\mathrm{R}}_{i}=\frac{\sum_{i=1}^{D} \mathrm{R}_{d i}}{D}, & d=1, \ldots, D
\end{array}
$$




$$
\begin{gathered}
\mathrm{R}_{d i}=\frac{C P_{d}-C P_{d-1}}{C P_{d-1}} \\
\mathrm{~V}_{\mathrm{R}}=\frac{\sum_{i=1}^{D}\left(\mathrm{R}_{d i}-\overline{\mathrm{R}}_{i}\right)^{2}}{D}, \quad d=1, \ldots, D
\end{gathered}
$$

where $\overline{\mathrm{R}}_{i}$ is the average return of the asset $i, \mathrm{~V}_{\mathrm{R}}$ is the variance of return for the asset $i, \mathrm{R}_{d i}$ is the daily return of the asset $i, C P_{d}$ is the closing price of the day, $C P_{d-1}$ is the closing price of the previous day, $i$ is the number of the assets, $d$ is the number of the days.

\section{Two Stage Portfolio Selection and Optimization Model}

Here, the authors have considered the SR model for portfolio optimization. For the selection of the portfolio, the mean of the return or variance of the return criteria has been taken in account. On such ground, there are two types of models based on selection criteria.

Model with mean criteria:

$$
\begin{aligned}
& \text { Descend } \quad \overline{\mathrm{R}}_{i}, \quad i=1, \ldots, N \\
& \text { Max SR } \\
& \text { Subject to } \sum_{i=1}^{N} w_{i}=1 \text {, } \\
& 0 \leqslant w_{i} \leqslant 1, \quad i=1, \ldots, N \text {. }
\end{aligned}
$$

Model with variance criteria:

$$
\begin{aligned}
& \text { Ascend } \mathrm{V}_{\mathrm{R}}, \quad i=1, \ldots, N \\
& \text { Max SR } \\
& \text { Subject to } \sum_{i=1}^{N} w_{i}=1, \\
& 0 \leqslant w_{i} \leqslant 1, \quad i=1, \ldots, N .
\end{aligned}
$$

The models presented above are of two stage. In the model (9), at first stage portfolio is selected with the mean criteria and then optimized with the maximization of SR as fitness function. On the other hand, in the model (10), initially the portfolio is selected with the variance criteria and then optimized with the maximization of SR as fitness function. In both the models, authors have considered the budget and restriction on short sale. Restriction on sale constraint means short sale is not allowed, it means that the proportion of an asset invested in the portfolio could not be negative or greater than 1 .

\section{Particle Swarm Optimization}

The PSO is supported by the population and their behaviour, which is inspired by the nature, like social behavior of bird flocking [27-29,32]. Here, each bird behaves as a particle in the swarm, each bird passes the information to the next bird, by this interacting behavior, in the form of group, they are able to execute very difficult tasks. The swarm initializes the particle 
position $x_{i}$ and velocity component $v_{i}^{k}$ as step size. The new position $x_{i}$ is being adjusted as given by equation (11) [25, 29]:

$$
x_{i}^{k+1}=x_{i}^{k}+v_{i}^{k+1}
$$

where as $v_{i}^{k+1}$, can be calculated and updated as:

$$
v_{i}^{k+1}=w v_{i}^{k}+c_{1} r_{1}\left[P_{\text {best }}-x_{i}^{k}\right]+c_{2} r_{2}\left[G_{\text {best }}-x_{i}^{k}\right]
$$

where $w$ is the inertia weight, $c_{1}$ and $c_{2}$ are the acceleration coefficients, $r_{1}$ and $r_{2}$ are the random numbers $r_{1}, r_{2} \in(0,1), P_{\text {best }}$ is the personal best position of particle $i$ called personal best, $G_{b e s t}$ is the best position of the particle $i$ called global best. To balance the global and local search, the large and small values of inertia weight $w$ play the significant role respectively. The $w, c_{1}$ and $c_{2}$ can be positive constant value or the positive decreasing linear function of the iteration index $k$, and are given as (13), (14) and (15) as described in [25, 29]:

$$
\begin{gathered}
w=w_{\text {max }}-\frac{w_{\text {max }}-w_{\text {min }}}{i t e r_{\text {max }}} \times k \\
c_{1}=c_{1 \text { max }}-\frac{c_{1 \text { max }}-c_{1 \text { min }}}{i t e r_{\text {max }}} \times k \\
c_{2}=c_{2 \max }-\frac{c_{2 \max }-c_{2 \min }}{i t e r_{\text {max }}} \times k
\end{gathered}
$$

The set values, i.e. $w_{\max }=0.9$ is the start of inertia weight, $w_{\min }=0.4$ is the end of inertia weight, $c_{1 \min }=c_{2 \min }=0$ are the start of acceleration coefficients, $c_{2 \max }=2.5, c_{2 \max }=4.5$ are the end of acceleration coefficients. Figure 1 shows the movement of particles in the optimization process $[25,29]$.

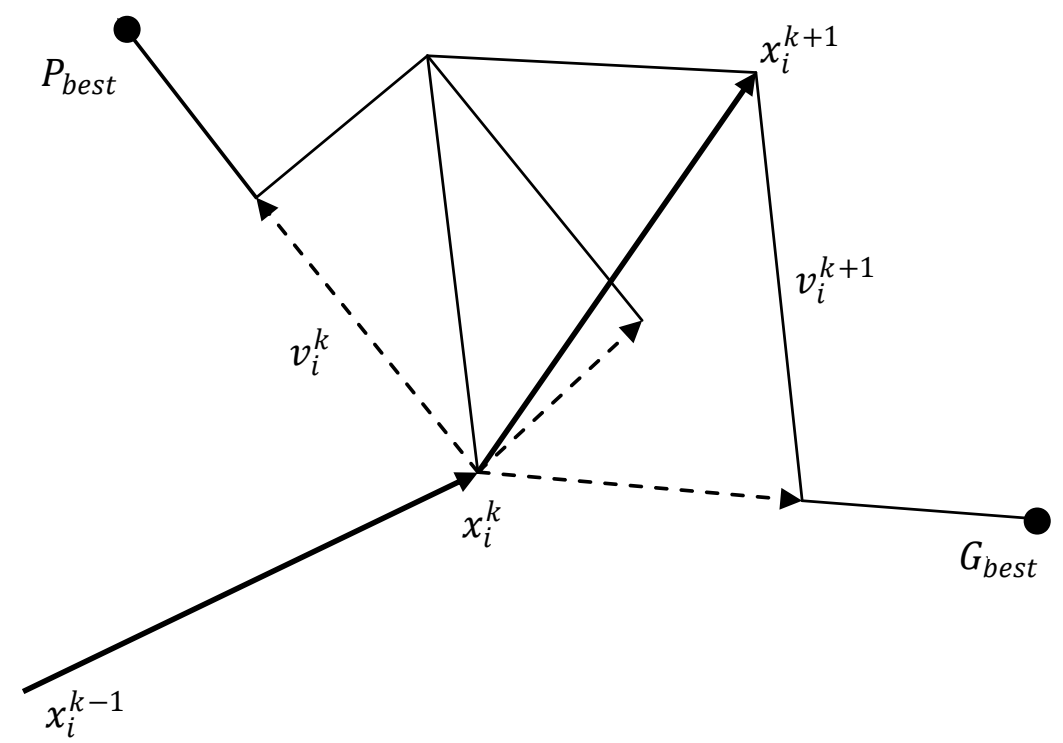

Figure 1: Particles Movement in the Process of Optimization.

Execution process for simple PSO is shown in Figure 2 which can be explained by the following steps $[25,29]$ : 
1. A population is randomly generated in the search space.

2. The initial velocity of each particle is randomly generated.

3. Objective function value for each particle is calculated.

4. The initial position of each particle is selected as its $P_{b e s t}$, and the best particle among the population is chosen as $G_{b e s t}$.

5. Particles move to new positions based on equations (11) and (12).

6. If a particle exceeds the allowed range, it is replaced by its previous position.

7. Objective function value for each particle is calculated.

8. $P_{\text {best }}$ and $G_{b e s t}$ are updated.

9. The stopping criteria are checked. If it is satisfied, the algorithm is terminated. Otherwise, Steps 5 to 8 are repeated. The maximum number of iterations can be used as stopping criteria to terminate the iterative process.

\subsection{Financial Tool Box-PSO (FTB-PSO)}

This FTB-PSO is a blend of FTB provided by MATLAB and the fundamental PSO algorithm. Here, it is to mention that some tasks have been performed in FTB besides the PSO. These tasks are portfolio establishment, calculation of mean and standard deviation of the portfolio, as well as, plot of the assets as particles of the portfolio. For the simulation in the FTB$\mathrm{PSO}$, it starts from loading the historical adjusted closed prices and the names assigned to the assets considered from SSE 50 index, in this case 21 days have been taken into account. In the next step, the data of prices is arranged, the mean, variance and covariance of the return are calculated. Next, the selection criteria have been defined on the basis of mean and variance of the return. Again the prices of 21 days are selected and the returns for the selected assets are obtained, the mean, variance and covariance of the returns for the selected assets are calculated, considering the budget constraint. The initial portfolio from FTB is built up and then the initial portfolio as scatter plot is displayed.

For PSO execution setup, its constants $w, c_{1}$ and $c_{2}$, also the bird steps as input iterations and the random matrices $R_{1}$ and $R_{2}$ are defined. The velocity is initialized as zero matrix, for position initialization the historical adjusted closed prices (as particles of the swarm) and their mean, variance and covariance have been considered. It is important to mention here that in the whole process of FTB-PSO the mean, variance and covariance are also updated with the update of particles. To check the accuracy, the level of sensitivity as input, is introduced here. Moreover, the iteration loop is started, the optimization process is run, the required matrices for best optimum solution are obtained, at each sensitivity level, the scatter plot is displayed as well as the position of the particle and the line plot for maximum SR is built.

Finally, the tabular values of maximum SR are obtained for the given sensitivity level. Besides, the maximum sharp ratio and its average in percent (\%) are calculated. In the end the scatter plot is displayed, line plot of average of maximum SR and surface plot are displayed 


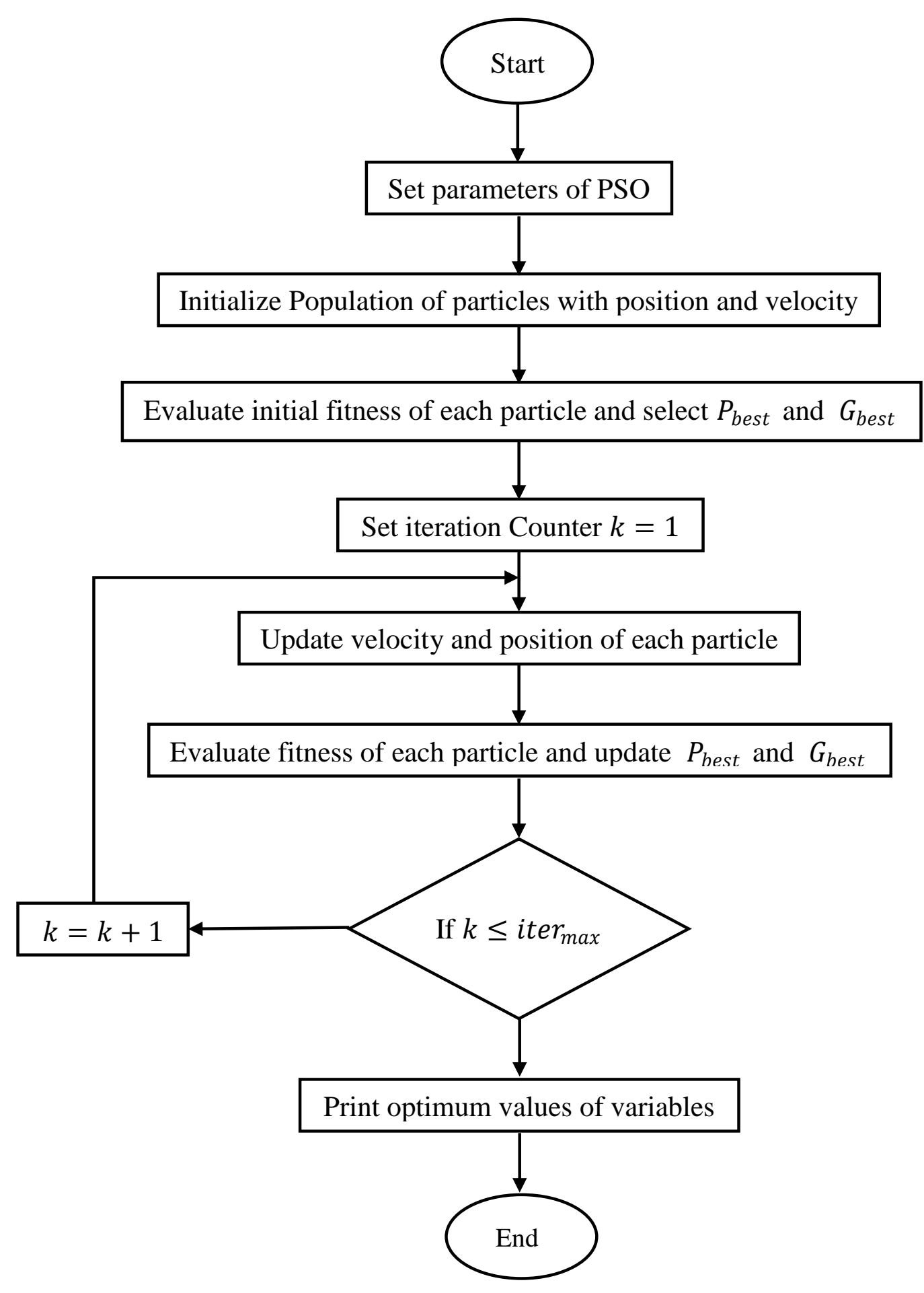

Figure 2: The Flowchart Depicting the General Algorithm of PSO 
to show the optimum level of the system for each considered case and sensitivity level for final stage of the system. The flow chart of this algorithm is given as under in Figure 3:

\section{Experiments and Discussion}

The simulation with FTB-PSO, as a Hybrid Particle Swarm Optimization (HPSO) for the portfolio optimization has been performed on two models for two stage portfolio selection and optimization. These models with two constraints, budget and restriction on short sale, have been considered for the different cases of collection of assets, like, 15 assets (A15), 30 assets (A30) and 50 assets (A50), in order to check the efficient diversification. To confirm the consistency of the FTB-PSO, the authors have performed the sensitivity analysis as 10 iterations. To update the portfolios, 200 iterations have been performed. The historical daily adjusted prices for the assets have been taken from the Shanghai Stock Exchange 50 Index (SSE 50 Index), as stated in preceding section 1.

Table 1 presents the numerical values of the maximum sharp ratios. These values are for three different number of assets collection A15, A30 and A50. Two different selection criteria, mean and variance have been taken into account. Apart from this, the sensitivity analysis have been done with 10 iterations. The tabular values show the rapid convergence of the system, especially, for diversified portfolios. Furthermore, it shows the importance of diversification, as it gets the higher value of maximum SRs for each criterion. From the tables given below, it can be extracted that the mean selection criteria would be better for consideration.

Table 1: Maximum Sharp Ratios

\begin{tabular}{|c|c|c|c|c|c|c|}
\hline \multirow{2}{*}{$\begin{array}{c}\text { No. of } \\
\text { Iterations }\end{array}$} & \multicolumn{5}{|c|}{ Maximum Sharp Ratios } \\
\cline { 2 - 7 } & \multicolumn{3}{|c|}{ Mean Criteria } & \multicolumn{3}{c|}{ Variance Criteria } \\
\cline { 2 - 7 } & $\mathrm{A} 15$ & $\mathrm{~A} 30$ & $\mathrm{~A} 50$ & $\mathrm{~A} 15$ & $\mathrm{~A} 30$ & A50 \\
\hline 01. & 1.191029 & 2.030254 & 2.918132 & 1.439870 & 1.578090 & 1.480579 \\
\hline 02. & 1.540944 & 1.880961 & 2.918349 & 0.992057 & 1.323320 & 1.852283 \\
\hline 03. & 1.532826 & 2.030254 & 2.918349 & 0.998544 & 1.681738 & 1.851099 \\
\hline 04. & 1.846623 & 1.880961 & 2.918349 & 0.998544 & 1.562444 & 1.850816 \\
\hline 05. & 1.687126 & 2.030254 & 2.918349 & 0.998544 & 1.323320 & 1.850900 \\
\hline 06. & 1.843232 & 1.880961 & 2.918349 & 0.998544 & 1.681738 & 1.850816 \\
\hline 07. & 1.532826 & 2.030254 & 2.918349 & 0.998544 & 1.562444 & 1.850900 \\
\hline 08. & 1.846623 & 1.880961 & 2.918349 & 0.998544 & 1.323320 & 1.850816 \\
\hline 09. & 1.687126 & 2.030254 & 2.918349 & 0.998544 & 1.681738 & 1.850900 \\
\hline 10. & 1.843232 & 1.880961 & 2.918349 & 0.998544 & 1.562444 & 1.850816 \\
\hline
\end{tabular}

Table 2 shows the maximum of the maximum SRs, for the collection of assets A15, A30 and A50 with mean and variance selection criteria in (\%).

Table 3 shows the average of the maximum SRs, for the collection of assets A15, A30 and A50 


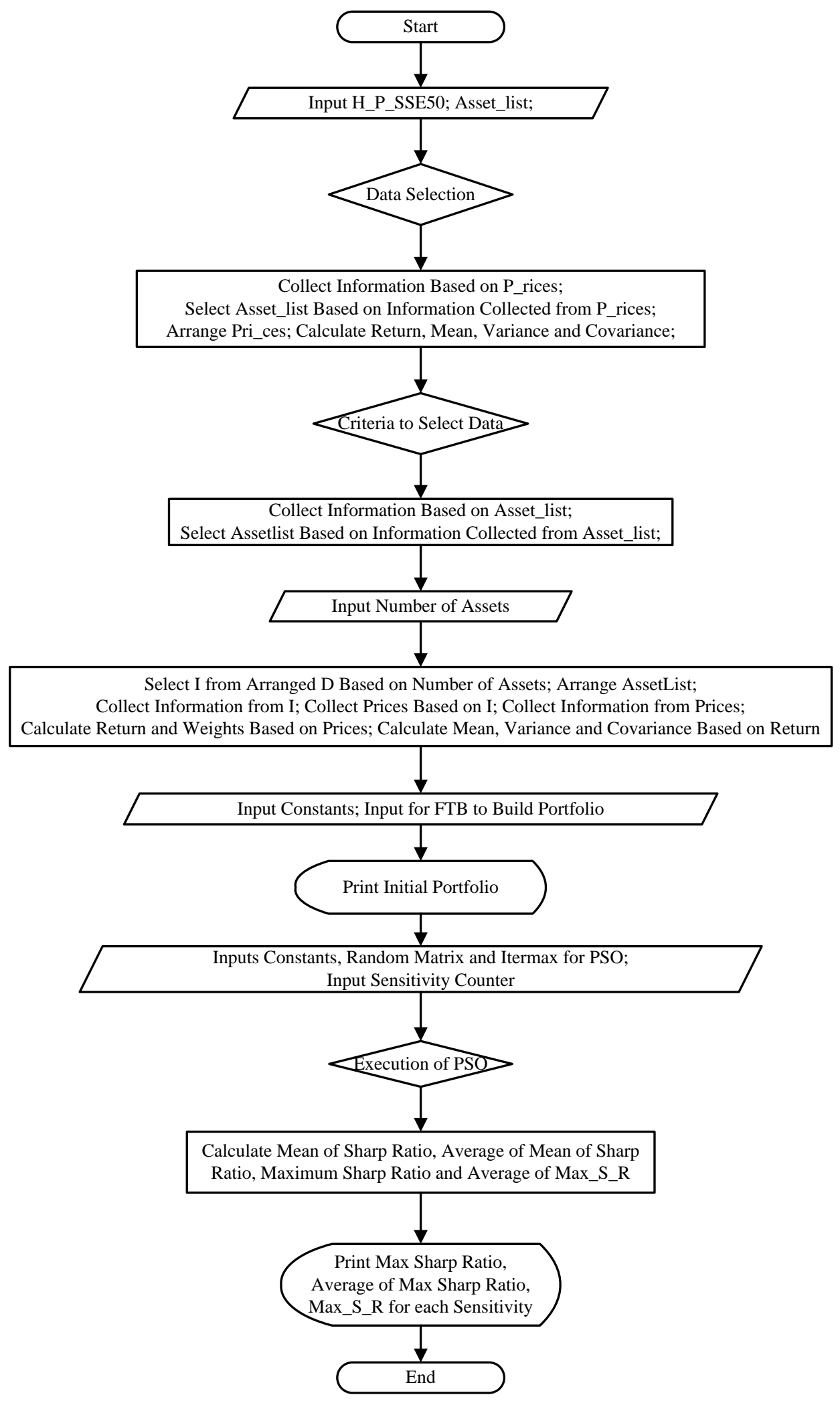

Figure 3: The Flowchart Depicting the General Algorithm of FTB-PSO. 
Table 2: Maximum Sharp Ratio (\%)

\begin{tabular}{|c|c|c|}
\hline Selection Criteria & No. of Assets & Sharp Ratio (\%) \\
\hline \multirow{3}{*}{ Mean } & A15 & 184.6623 \\
\cline { 2 - 3 } & A30 & 203.0254 \\
\cline { 2 - 3 } & A50 & 291.8349 \\
\hline \multirow{3}{*}{ Variance } & A15 & 143.9870 \\
\cline { 2 - 3 } & A30 & 168.1738 \\
\cline { 2 - 3 } & A50 & 185.2283 \\
\hline
\end{tabular}

with mean and variance selection criteria in (\%).

Table 3: Average of Maximum Sharp Ratio (\%)

\begin{tabular}{|c|c|c|}
\hline Selection Criteria & No. of Assets & Sharp Ratio (\%) \\
\hline \multirow{3}{*}{ Mean } & A15 & 165.5159 \\
\cline { 2 - 3 } & A30 & 195.5608 \\
\cline { 2 - 3 } & A50 & 291.8327 \\
\hline \multirow{3}{*}{ Variance } & A15 & 104.2028 \\
\cline { 2 - 3 } & A30 & 152.8060 \\
\cline { 2 - 3 } & A50 & 181.3993 \\
\hline
\end{tabular}

Figures 4, 6, 8, 10, 12 and 14 are the scattered (a) and surface (b) plots, for A15, A30 and A50 portfolios with mean and variance selection criteria respectively. The peaks in these figures show the convergence of the system for maximization of SRs.

Figures 5, 7, 9, 11, 13 and 15 are the line (a) and surface (b) plot for each iterations and all sensitivity analysis respectively, where as A15, A30 and A50 portfolios with mean and variance selection criteria have been considered. The line and peaks represent the maximum value of the SRs at an iteration, which shows the convergence of the system for maximization of SRs.

\section{Conclusion}

In this paper, the authors proposed and developed two simple models for optimization with the combination of mean, variance and SR of returns. The efficient financial tool box (FTB)particle swarm optimization (PSO), i.e. FTB-PSO algorithm has been developed. In this regard, the authors have developed, two new efficient frontier, with the single constraint, as two stage portfolio optimization models. Furthermore, the basic principle of investment, the diversification of the asset is validated. It is also concluded that higher the diversification, higher the profit. This study will contribute significantly for an individual investor, in the financial market, institutions and banks etc. 

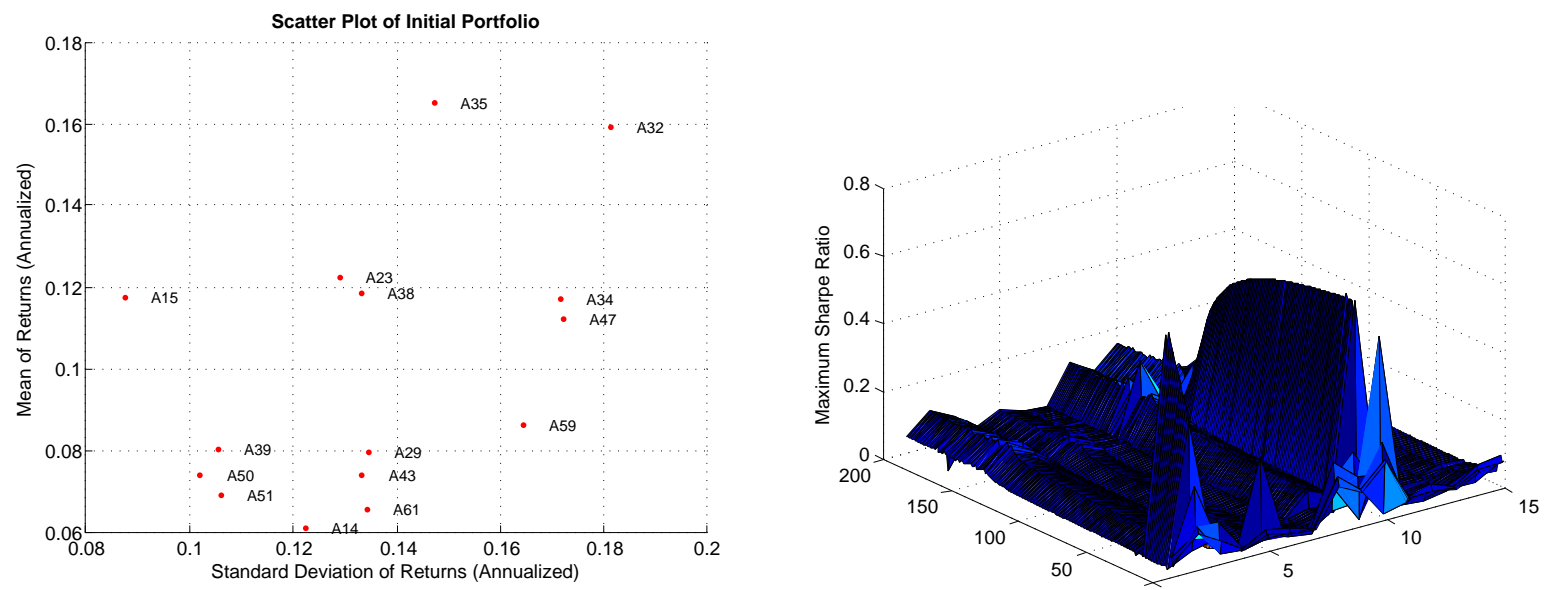

Figure 4: Scattered Initial and Surface Plot for A15 with Mean Selection Criteria
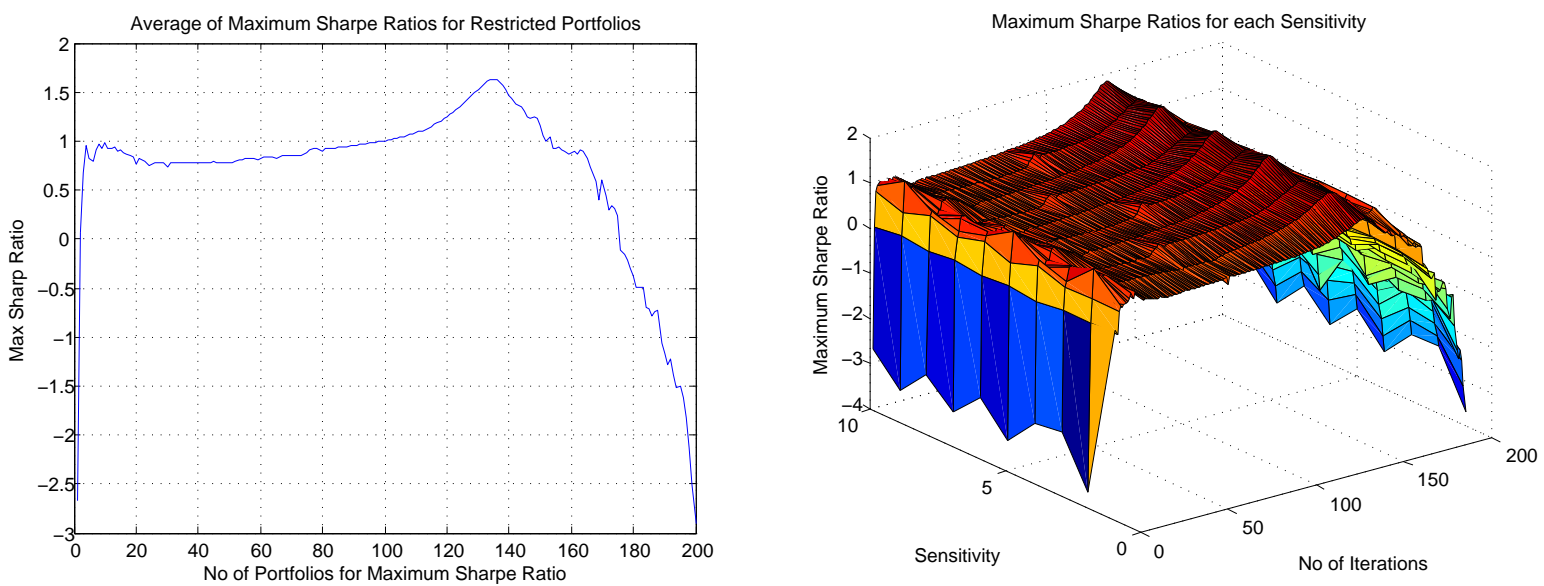

Figure 5: Plots for Each Iterations of A15 with Mean Selection Criteria
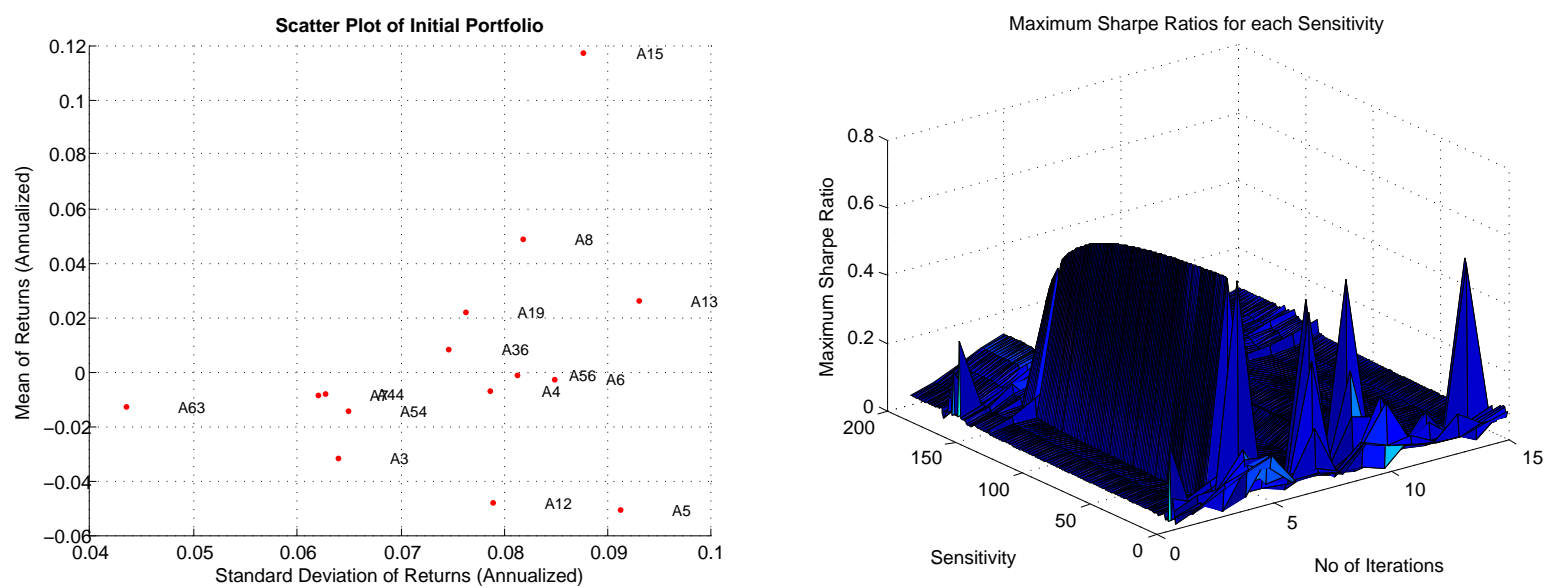

Figure 6: Scattered Initial and Surface Plot for A15 with Variance Selection Criteria 

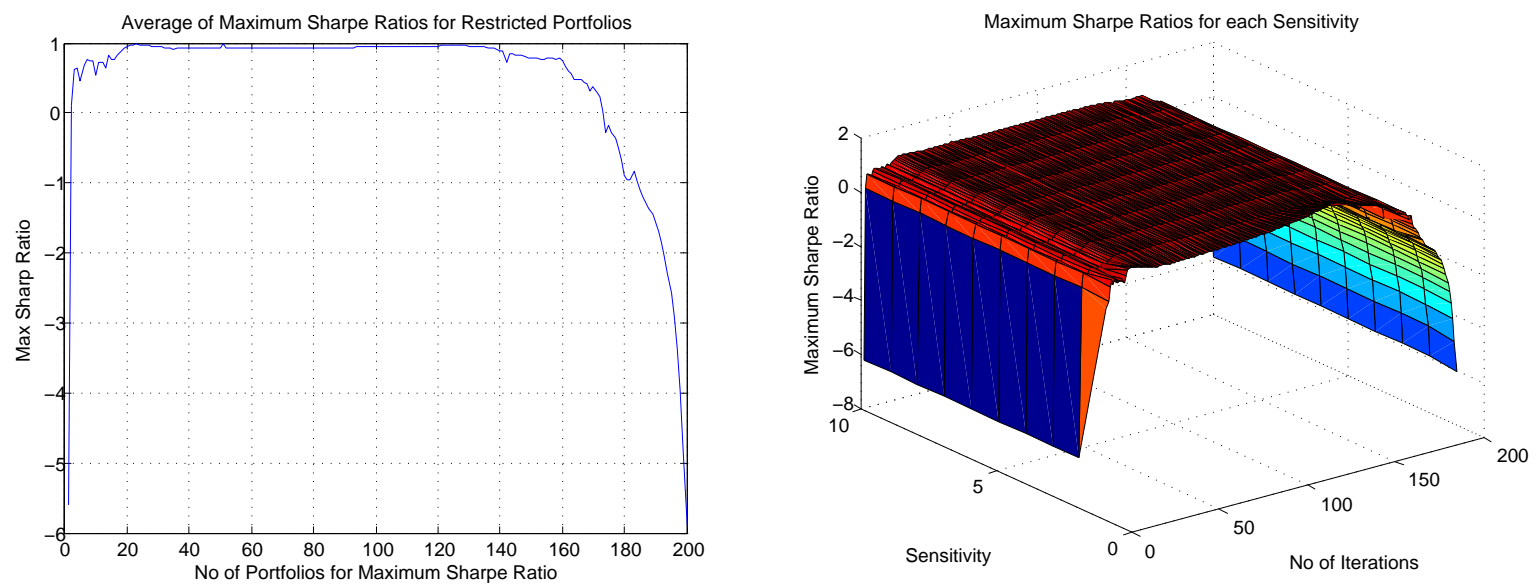

Figure 7: Plots for Each Iterations of A15 with Variance Selection Criteria
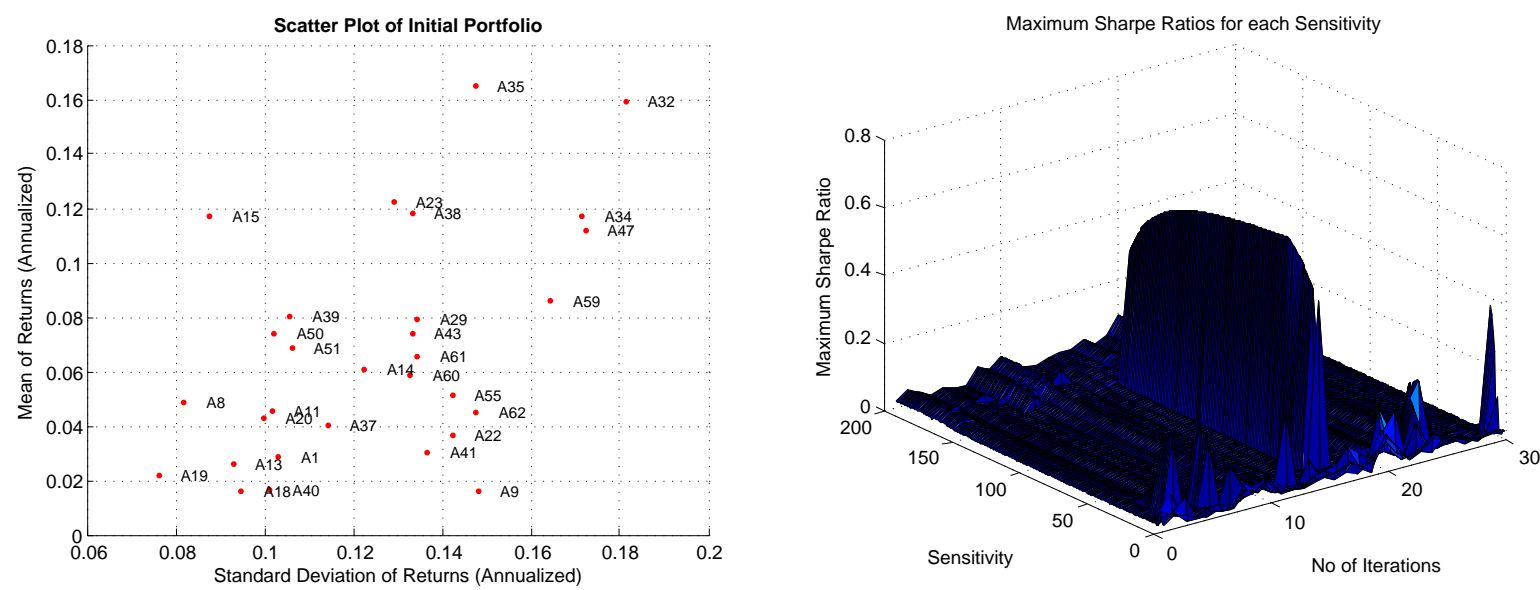

Figure 8: Scattered Initial and Surface Plot for A30 with Mean Selection Criteria
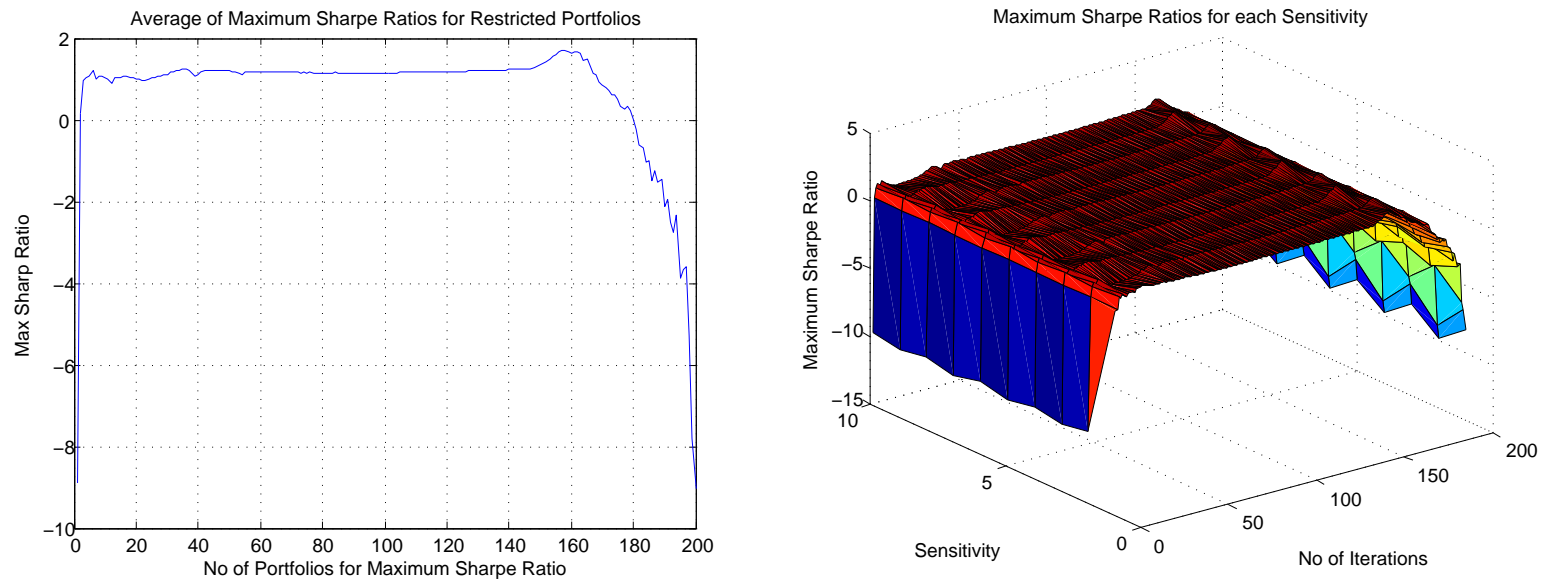

Figure 9: Plots for Each Iterations of A30 with Mean Selection Criteria 

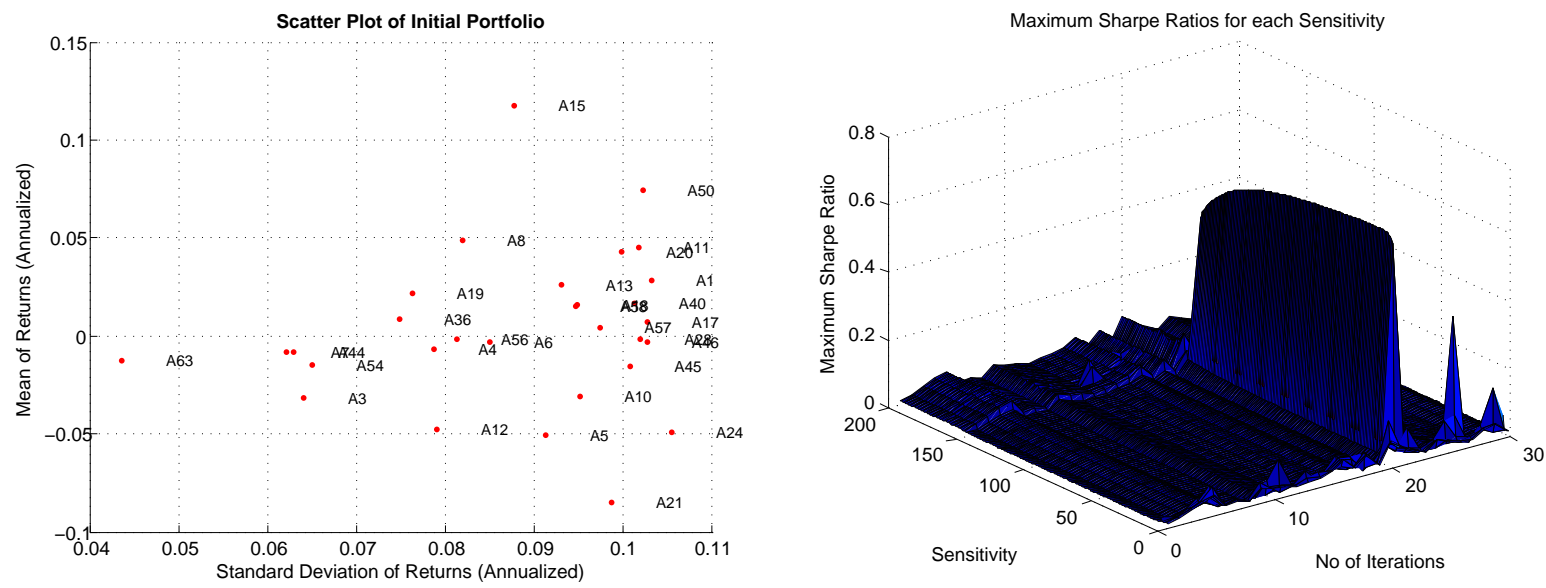

Figure 10: Scattered Initial and Surface Plot for A30 with Variance Selection Criteria
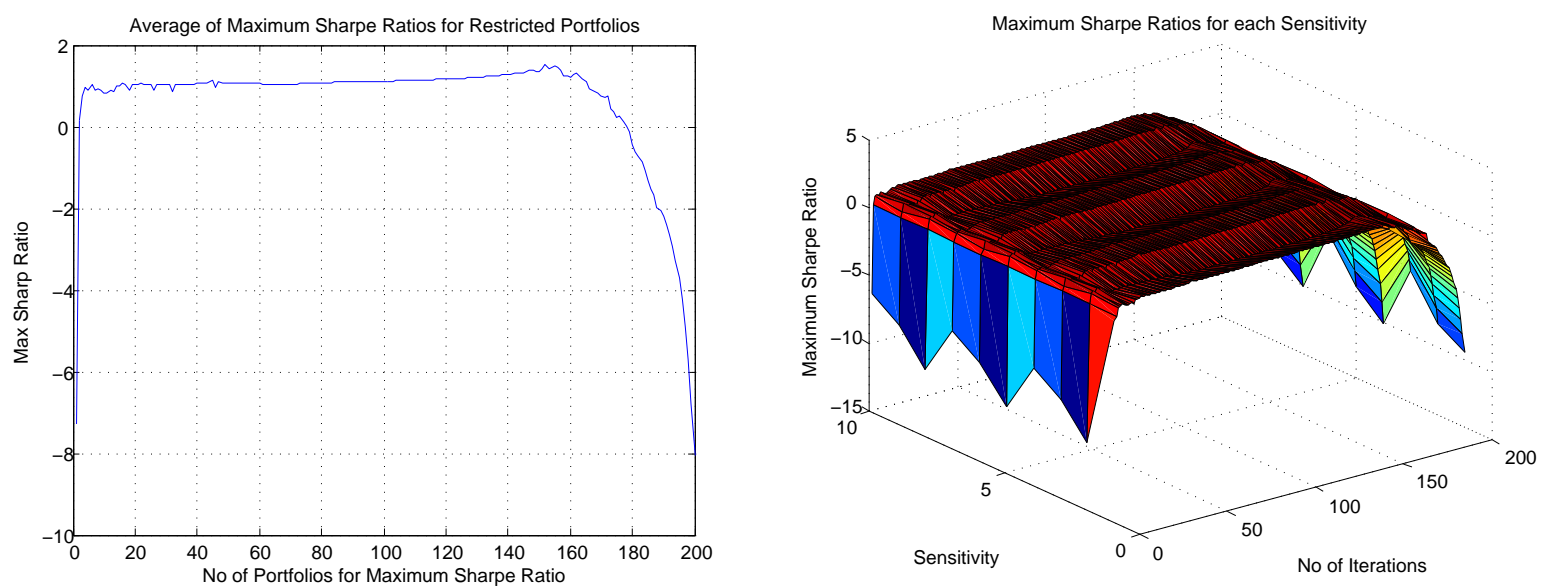

Figure 11: Plots for Each Iterations of A30 with Variance Selection Criteria
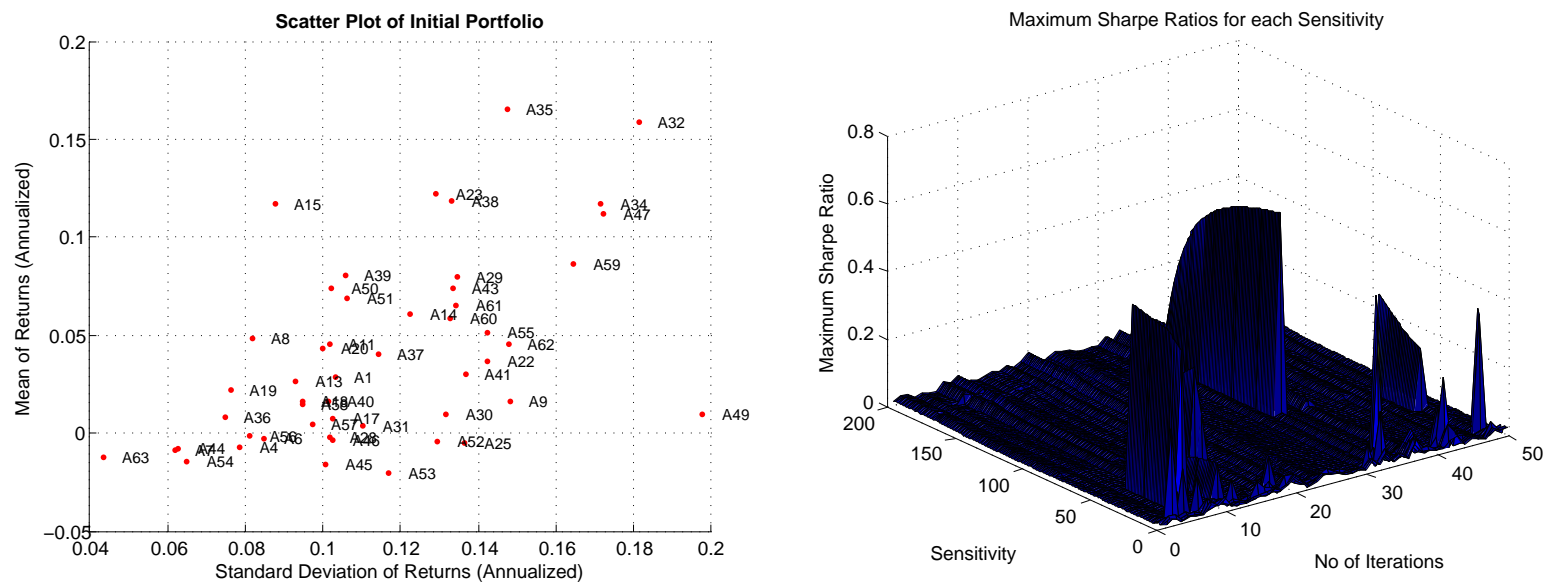

Figure 12: Scattered Initial and Surface Plot for A50 with Mean Selection Criteria 

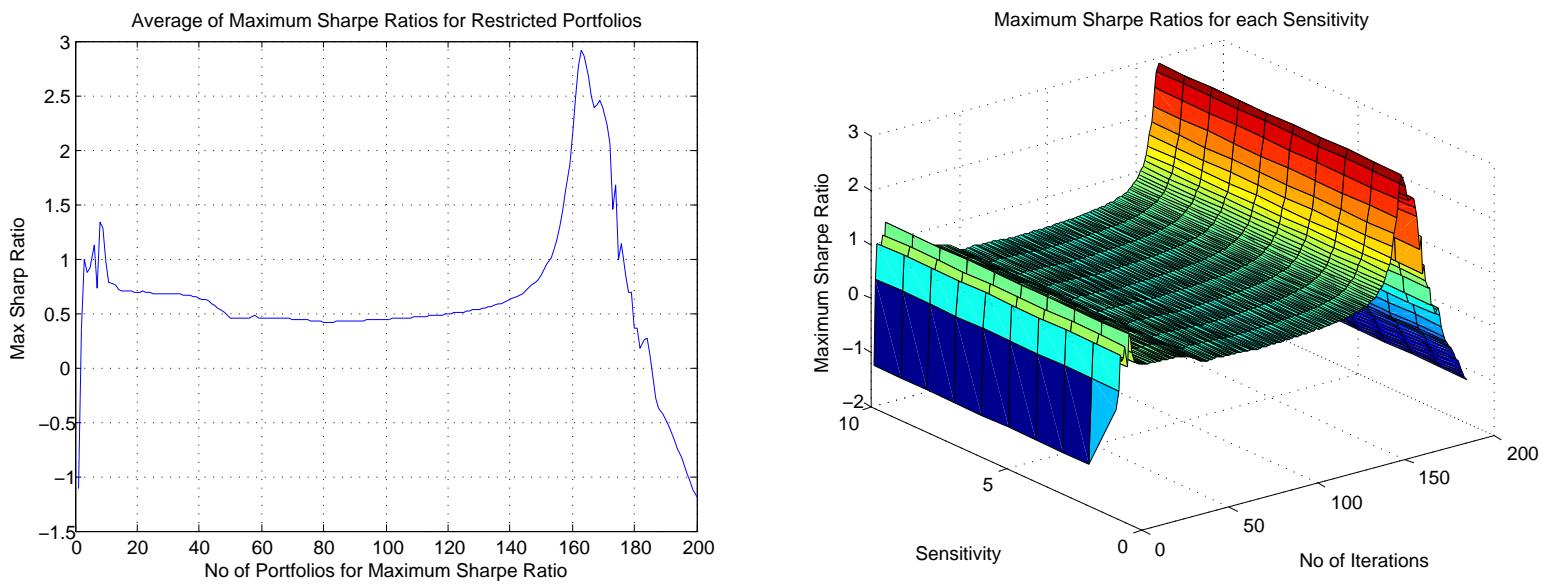

Figure 13: Plots for Each Iterations of A50 with Mean Selection Criteria.

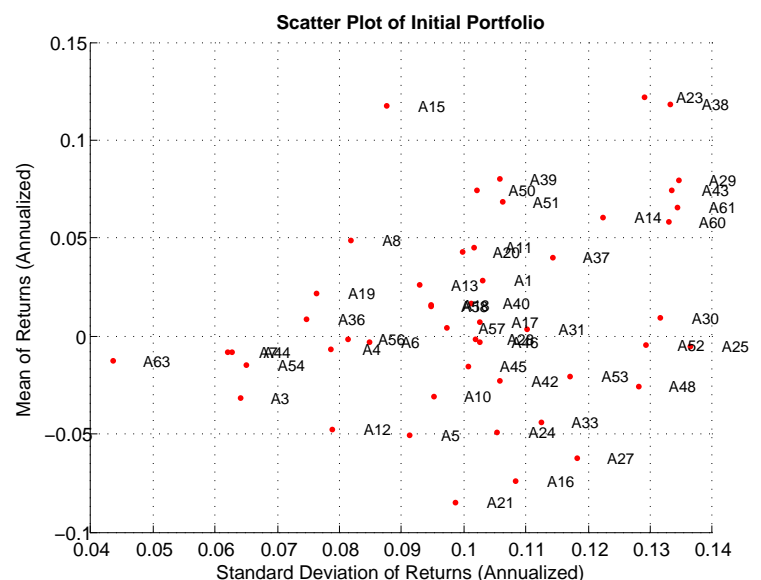

Maximum Sharpe Ratios for each Sensitivity

Figure 14: Scattered Initial and Surface Plot for A50 with Variance Selection Criteria
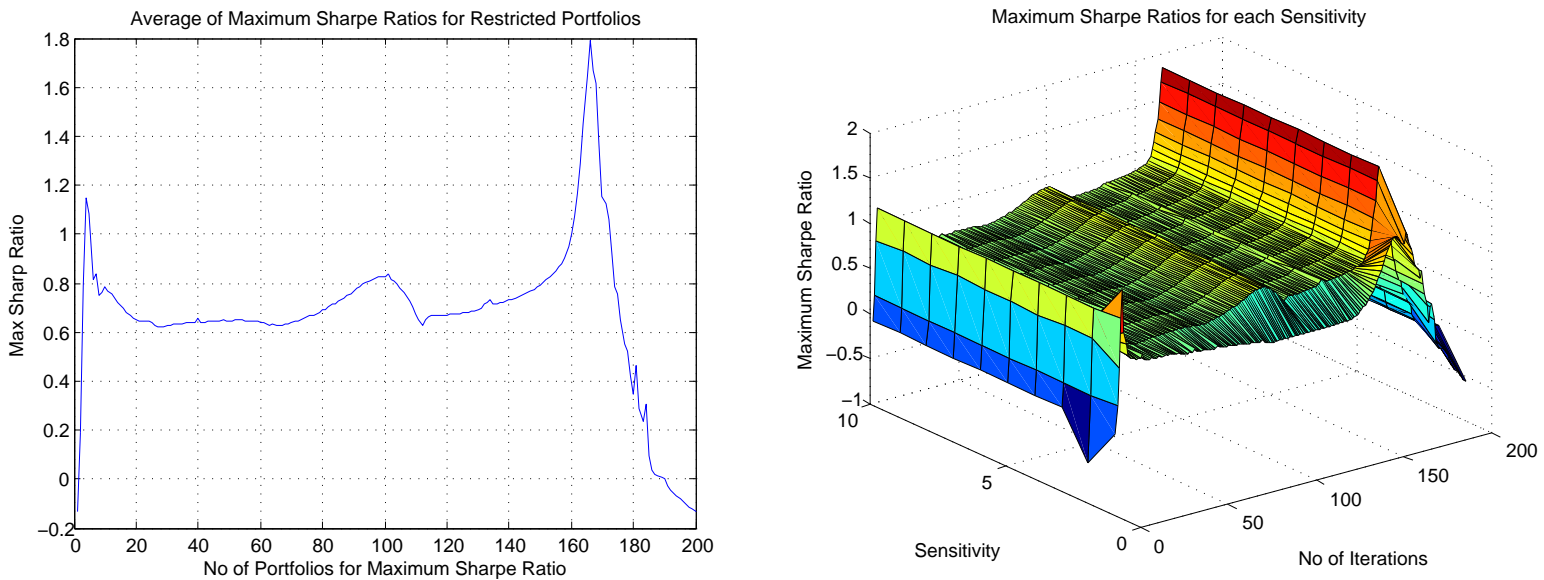

Figure 15: Plots for Each Iterations of A50 with Variance Selection Criteria 
The inclusion of some other constraints to the model developed, like short sale allowance, transaction cost, liquidity of the assets and minimum lot as constraints, are some recommendations for the future research. Apart from this, the study can be further carried out with some other meta-heuristic techniques as well.

\section{Acknowledgments}

The author, particularly, Kashif Bin Zaheer, is grateful to Federal Urdu University of Arts, Sciences \& Technology (FUUAST) Karachi, Pakistan, for the financial support, under the project Faculty Development Program (FDP) funded by Higher Education Commission (HEC) of Pakistan.

\section{References}

[1] Markoviz, H. Portfolio Selection: Efficient Diversification of Investments. New York: John Wiley \& Sons, Inc. 1959.

[2] Thirimanna, T. H. S. R., Tilakartane, C., Mahakalanda, I. and Pathirathne, L. Portfolio selection using cointegration and modern portfolio theory: An application to the colombo stock exchange. Matematika. 2013. 29: 195-202.

[3] Choon, P. T. and Wei, X. L. Universal portfolio using the best current-run parameter. Matematika. 2013. 29: 89-94.

[4] Chen, K. C., Ying, T. S. and Bahar, A. Probability distribution of returns in heston model and hurst exponent estimation for index prices of ftse bursa malaysia klci. Matematika. 2014. 30: $44-58$.

[5] Sagir, A. M. and Sathasivan, S. The use of artificial neural network and multiple linear regressions for stock market forecasting. Matematika. 2017. 33(1): 1-10.

[6] Chen, W. and Zhang, W.-G. The admissible portfolio selection problem with transaction costs and an improved pso algorithm. Physica A: Statistical Mechanics and its Applications. 2010. 389(10): 2070-2076.

[7] Golmakani, H. R. and Fazel, M. Constrained portfolio selection using particle swarm optimization. Expert Systems with Applications. 2011. 38(7): 8327-8335.

[8] Deng, G.-F., Lin, W.-T. and Lo, C.-C. Markowitz-based portfolio selection with cardinality constraints using improved particle swarm optimization. Expert Systems with Applications. 2012. 39(4): 4558-4566.

[9] Gao, J. and Chu, Z. An improved particle swarm optimization for the constrained portfolio selection problem. In Computational Intelligence and Natural Computing, 2009. CINC'09. International Conference on. IEEE. 2009. vol. 1. 518-522.

[10] Qin, Z. Mean-variance model for portfolio optimization problem in the simultaneous presence of random and uncertain returns. European Journal of Operational Research. 2015. 245(2): 480-488. 
[11] Lwin, K., Qu, R. and Kendall, G. A learning-guided multi-objective evolutionary algorithm for constrained portfolio optimization. Applied Soft Computing. 2014. 24: 757-772.

[12] Steinbach, M. C. Markowitz revisited: Mean-variance models in financial portfolio analysis. SIAM review. 2001. 43(1): 31-85.

[13] Krink, T. and Paterlini, S. Multiobjective optimization using differential evolution for real-world portfolio optimization. Computational Management Science. 2011. 8(1): 157179.

[14] Di Gaspero, L., Di Tollo, G., Roli, A. and Schaerf, A. Hybrid local search for constrained financial portfolio selection problems. Integration of AI and OR Techniques in Constraint Programming for Combinatorial Optimization Problems. 2007: 44-58.

[15] Chang, T.-J., Meade, N., Beasley, J. E. and Sharaiha, Y. M. Heuristics for cardinality constrained portfolio optimisation. Computers $\& 3$ Operations Research. 2000. 27(13): 12711302.

[16] Duan, Y. C. A multi-objective approach to portfolio optimization. Rose-Hulman Undergraduate Mathematics Journal. 2007. 8(1): 12.

[17] Sharpe, W. F. A simplified model for portfolio analysis. Management science. 1963. 9(2): $277-293$.

[18] Bailey, D. H. and Lopez de Prado, M. The sharpe ratio efficient frontier. 2012.

[19] Farinelli, S., Ferreira, M., Rossello, D., Thoeny, M. and Tibiletti, L. Beyond sharpe ratio: Optimal asset allocation using different performance ratios. Journal of Banking $\& 3$ Finance. 2008. 32(10): 2057-2063.

[20] Gao, X. and Chan, L. An algorithm for trading and portfolio management using q-learning and sharpe ratio maximization. In Proceedings of the international conference on neural information processing. 2000. 832-837.

[21] Deng, G., Dulaney, T., McCann, C. and Wang, O. Robust portfolio optimization with value-at-risk-adjusted sharpe ratios. Journal of Asset Management. 2013. 14(5): 293-305.

[22] Yang, C. and Simon, D. A new particle swarm optimization technique. In Systems Engineering, 2005. ICSEng 2005. 18th International Conference on. IEEE. 2005. 164-169.

[23] Vinod, H. D. and Morey, M. R. A double sharpe ratio. 1999.

[24] Choey, M. and Weigend, A. S. Nonlinear trading models through sharpe ratio maximization. International Journal of Neural Systems. 1997. 8(04): 417-431.

[25] Zhu, H., Wang, Y., Wang, K. and Chen, Y. Particle swarm optimization (pso) for the constrained portfolio optimization problem. Expert Systems with Applications. 2011. 38(8): 10161-10169. 
[26] Mokhtar, M., Shuib, A. and Mohamad, D. Mathematical programming models for portfolio optimization problem: A review. International Journal of Social, Management, Economics and Business Engineering. 2014. 8(2): 443-450.

[27] Kennedy, J. and Eberhart, R. C. The particle swarm: social adaptation in informationprocessing systems. In New ideas in optimization. McGraw-Hill Ltd., UK. 1999. 379-388.

[28] Kennedy, J. Particle swarm optimization. In Encyclopedia of machine learning. Springer. 760-766. 2011.

[29] Maleki, A., Ameri, M. and Keynia, F. Scrutiny of multifarious particle swarm optimization for finding the optimal size of a pv/wind/battery hybrid system. Renewable Energy. 2015. 80: $552-563$.

[30] He, Q. and Wang, L. A hybrid particle swarm optimization with a feasibility-based rule for constrained optimization. Applied mathematics and computation. 2007. 186(2): 1407-1422.

[31] Prasain, H., Jha, G. K., Thulasiraman, P. and Thulasiram, R. A parallel particle swarm optimization algorithm for option pricing. In Parallel $\mathscr{E}$ Distributed Processing, Workshops and Phd Forum (IPDPSW), 2010 IEEE International Symposium on. IEEE. 2010. 1-7.

[32] Eberhart, R. and Kennedy, J. A new optimizer using particle swarm theory. In $\mathrm{Mi}$ cro Machine and Human Science, 1995. MHS'95., Proceedings of the Sixth International Symposium on. IEEE. 1995. 39-43. 